\title{
Comparison of the Diagnostic Ability of Endoscopic Ultrasonography and Abdominopelvic Computed Tomography in the Diagnosis of Gastric Subepithelial Tumors
}

\author{
Sang Yoon Kim¹, Ki-Nam Shim¹, Joo-Ho Lee ${ }^{2}$, Ji Young Lim', Tae Oh Kim¹, A. Reum Choe', Chung Hyun Tae', Hye-Kyung Jung', \\ Chang Mo Moon ${ }^{1}$, Seong-Eun Kim ${ }^{1}$ and Sung-Ae Jung ${ }^{1}$
}

Department of ${ }^{1}$ Internal Medicine, ${ }^{2}$ Surgery, College of Medicine, Ewha Womans University, Seoul, Korea

Background/Aims: Endoscopic ultrasonography (EUS) is the most efficient imaging modality for gastric subepithelial tumors (SETs). However, abdominopelvic computed tomography (APCT) has other advantages in evaluating the characteristics, local extension, or invasion of SETs to adjacent organs. This study aimed to compare the diagnostic ability of EUS and APCT based on surgical histopathology results.

Methods: We retrospectively reviewed data from 53 patients who underwent both EUS and APCT before laparoscopic wedge resection for gastric SETs from January 2010 to December 2017 at a single institution. On the basis of histopathology results, we assessed the diagnostic ability of the 2 tests.

Results: The overall accuracy of EUS and APCT was $64.2 \%$ and $50.9 \%$, respectively. In particular, the accuracy of EUS vs. APCT for the diagnosis of gastrointestinal stromal tumors (GISTs), leiomyomas, and ectopic pancreas was $83.9 \%$ vs. $74.2 \%$, $37.5 \%$ vs. $0.0 \%$, and $57.1 \%$ vs. $14.3 \%$, respectively. Most of the incorrect diagnoses with EUS involved hypoechoic lesions originating in the fourth echolayer, with the most common misdiagnosed lesions being GISTs mistaken for leiomyomas and vice versa.

Conclusions: APCT showed a lower overall accuracy than EUS; however, APCT remains a useful modality for malignant/potentially malignant gastric SETs. Clin Endosc 2019;52:565-573

Key Words: Abdominopelvic computed tomography; Diagnosis; Endoscopic ultrasonography; Subepithelial tumor

\section{INTRODUCTION}

Gastric subepithelial tumors (SETs) are identified as masslike lesions covered with normal mucosa from the gastrointestinal (GI) tract. ${ }^{1,2}$ The prevalence of SETs in the GI tract is not well known. However, the detection rate of suspected gastric

Received: January 1, 2019 Revised: March 17, 2019

Accepted: April 10, 2019

Correspondence: Ki-Nam Shim

Department of Internal Medicine, College of Medicine, Ewha Womans University, 260 Gonghang-daero, Gangseo-gu, Seoul 07804, Korea

Tel: +82-2-2650-2632, Fax: +82-2-2655-2076, E-mail: shimkn@ewha.ac.kr

ORCID: https://orcid.org/0000-0003-4004-6292

(cc) This is an Open Access article distributed under the terms of the Creative Commons Attribution Non-Commercial License (http://creativecommons.org/ licenses/by-nc/3.0) which permits unrestricted non-commercial use, distribution, and reproduction in any medium, provided the original work is properly cited.
SETs with esophagogastroduodenoscopy (EGD) has previously been reported to be approximately $0.4 \% .^{3}$ In general, SETs are incidentally detected during endoscopy or identified using abdominopelvic computed tomography (APCT) without the presentation of specific symptoms. ${ }^{4}$ Because of recent advances in endoscopy and improvement in the quality of CT imaging, the detection of gastric SETs is expected to considerably increase. Gastric SETs include various types of tumors, ranging from benign to malignant. ${ }^{1,5}$ Potentially malignant or malignant gastric SETs include gastrointestinal stromal tumors (GISTs), lymphomas, carcinoid tumors, and glomus tumors. ${ }^{1}$ These SETs should be differentiated for accurate diagnosis and appropriate treatment.

Currently, endoscopic ultrasonography (EUS) is the most sensitive imaging modality for the evaluation of gastric SETs. ${ }^{6}$ Owing to its high spatial resolution, EUS is able to provide 
information about the layer of origin, size, internal echogenicity, and echotexture of a tumor. ${ }^{6,7}$ On the other hand, APCT, which is a less invasive modality, is widely conducted for abdominal assessments in clinical practice. ${ }^{8}$ Recent studies have reported that the accuracy of APCT in detecting gastric disease has increased with the improvement in CT imaging quality. ${ }^{4,89}$ APCT has unique advantages in the characterization of SETs based on CT attenuation values and evaluation of lymph node status; ${ }^{10-12}$ however, it is unable to determine the layer of origin, which is an important factor in presumptive diagnosis. ${ }^{9,13}$ Therefore, APCT is expected to play a more relevant role in the diagnosis of gastric SETs in the future. To date, the diagnostic ability of EUS and APCT with respect to gastric SETs has not been adequately compared, with only a few previous studies having reported the accuracy of APCT based on EUS findings as the standard of reference. ${ }^{9,13,14}$ This study therefore aimed to compare the diagnostic ability of EUS and APCT and to assess whether they are satisfactory modalities for diagnosing gastric SETs based on surgical histopathology results. In addition, cases of misdiagnosis with EUS were analyzed, and the causes of these misdiagnoses were identified.

\section{MATERIALS AND METHODS}

\section{Study subjects}

We retrospectively investigated 53 patients who underwent laparoscopic wedge resection for suspected gastric SETs from January 2010 to December 2017 at Ewha Womans University Mokdong Hospital. All enrolled patients underwent both EGD and APCT for the evaluation of gastric SETs. On the basis of surgical histopathology results, we reviewed the presumptive diagnosis of preoperative EUS and APCT. The gastric SET cases were divided into 2 groups based on the histopathology results: benign and malignant/potentially malignant. Benign SETs mainly included leiomyomas, ectopic pancreas, schwannomas, lipomas, gastric duplication cysts (GDCs), and adenomyomas, whereas malignant/potentially malignant SETs included GISTs and lymphomas.

\section{Endoscopic ultrasonography}

EUS was performed by 2 investigators, each with $>15$ years of experience in gastroenterology. When a gastric SET was detected using EUS, the location (antrum, angle, body, cardia, or fundus), size, echotexture (homogeneous or heterogeneous), internal echogenicity (hypoechoic, hyperechoic, anechoic, or mixed), and layer of origin (mucosa, muscularis mucosa, submucosa, muscularis propria, or serosa) were described. Depending on the size and location, radial EUS (GF-UE260; Olympus Medical Systems Co., Tokyo, Japan) or a catheter probe (Olympus GIF 2T 240 with UM-3R) was used for 29 and 24 of the gastric SETs, respectively. During EUS, distilled water was injected through the endoscope, if needed.

\section{Abdominopelvic computed tomography}

APCT images were interpreted by 2 board-certified abdominal radiologists independently of the EUS results. They analyzed the size, location (antrum, angle, body, cardia, or fundus), enhancement degree (difference in Hounsfield units $[\mathrm{HU}]$ between the gastric wall and the lumen), enhancement pattern, and contour of the gastric SETs and provided an initial diagnosis. When the type of gastric SET was difficult to determine on APCT images owing to limited resolution capability, the tumor was classified as a possible gastric SET. APCT images were obtained using 64-section CT (Somatom Sensation 64; SIEMENS, München, Germany; $n=42$ ) and 128-section CT (Somatom Definition Flash; SIEMENS; $n=11$ ). The parameters for both 64-section and 128-section CT were as follows: slice thickness of $3 \mathrm{~mm}$, peak voltage of $120 \mathrm{kVp}$, and injection of $120 \mathrm{~mL}$ of nonionic contrast material (Bonorex 350 ) at a rate of $2.0 \mathrm{~mL} / \mathrm{s}$. The tube currents of 64 -section and 128-section CT were 170 and $150 \mathrm{~mA}$, respectively. To ensure optimal conditions for gastric distension, all patients fasted for at least $8 \mathrm{~h}$.

\section{Assessment of diagnostic ability}

We calculated the diagnostic ability (accuracy, sensitivity, specificity, positive predictive value [PPV], and negative predictive value [NPV]) of EUS and APCT for gastric SETs based on the surgical histopathology results, which were used to definitively diagnose the SETs.

\section{Statistical analyses}

The differences between benign gastric SETs and malignant/potentially malignant gastric SETs were analyzed using Student's $t$-tests or Mann-Whitney $U$-tests and chi-squared tests. Student's $t$-tests and chi-squared tests were used for continuous and categorical variables, respectively. Median (interquartile range) and nonparametric tests (Mann-Whitney $U$-tests) were used because the tumor size distribution was skewed. Statistical analysis was conducted using SPSS 21.0 for Windows (SPSS Inc., Chicago, IL, USA). A 2-tailed $p$-value of $<0.05$ was considered statistically significant.

\section{Ethics statement}

This study was approved by the Institutional Review Board of Ewha University Mokdong Hospital (approval no. 2018-08052-001). Written informed consent was not obtained from the patients because this study had a retrospective design. The records of the patients were anonymized and de-identified be- 
fore the analysis to protect their privacy. This study followed the Declaration of Helsinki.

Table 1. Histopathology Results of Gastric Subepithelial Tumors

\begin{tabular}{lc}
\hline Histopathology results & $\boldsymbol{n}(\%)$ \\
\hline Total & $53(100)$ \\
Malignant/Potentially malignant & $31(58.5)$ \\
GIST & $1(1.9)$ \\
Lymphoma & \\
Benign & $8(15.1)$ \\
Leiomyoma & $7(13.2)$ \\
Ectopic pancreas & $3(5.6)$ \\
Schwannoma & $1(1.9)$ \\
Lipoma & $1(1.9)$ \\
Gastric duplication cyst & $1(1.9)$ \\
Adenomyoma & \\
\hline
\end{tabular}

GIST, gastrointestinal stromal tumor.

\section{RESULTS}

\section{Histopathology results for gastric subepithelial tu-} mors

A total of 53 consecutive patients who underwent both EUS and APCT before laparoscopic wedge resection for gastric SETs from January 2010 to December 2017 were reviewed. The histopathology results are summarized in Table 1. Of 32 patients (60.4\%) with malignant/potentially malignant gastric SETs, GIST was identified in 31 patients (58.5\%) and lymphoma in 1 patient (1.9\%). Of 21 patients (39.6\%) with benign gastric SETs, leiomyoma was confirmed in 8 patients (15.1\%), ectopic pancreas in 7 patients (13.2\%), schwannoma in 3 patients (5.6\%), lipoma in 1 patient (1.9\%), GDC in 1 patient (1.9\%), and adenomyoma in 1 patient (1.9\%).

\section{Clinical characteristics of the study patients}

Table 2 shows the characteristics of the study patients. Their mean age was $60.4 \pm 14.9$ years, and $37.7 \%$ were men. The

Table 2. Clinical Characteristics of the Study Subjects

\begin{tabular}{|c|c|c|c|c|}
\hline Characteristics & Total & Benign & Malignant/Potentially malignant & $p$-value \\
\hline Age, $\mathrm{yr}^{\mathrm{a}}$ & $60.4 \pm 14.9$ & $52.7 \pm 14.1$ & $65.4 \pm 13.3$ & 0.002 \\
\hline Sex, $n(\%)$ & & & & 0.409 \\
\hline Men & $20(37.7)$ & $6(28.6)$ & $14(43.75)$ & \\
\hline Women & $33(62.3)$ & $15(71.4)$ & $18(56.25)$ & \\
\hline Size on histopathology $(\mathrm{cm})^{\mathrm{b})}$ & $3.0(2.5-4.5)$ & $3.0(2.5-4.5)$ & $3.0(2.4-4.0)$ & 0.848 \\
\hline Location, $n(\%)$ & & & & $<0.001$ \\
\hline Cardia & $7(13.2)$ & $6(28.6)$ & $1(3.1)$ & \\
\hline Fundus & $5(9.4)$ & $0(0.0)$ & $5(15.6)$ & \\
\hline Body & $33(62.3)$ & $8(38.1)$ & $25(78.1)$ & \\
\hline Antrum & $8(15.1)$ & $7(33.3)$ & $1(3.1)$ & \\
\hline Layer of origin, $n(\%)$ & & & & 0.097 \\
\hline Second & $2(3.5)$ & $2(9.5)$ & $0(0.0)$ & \\
\hline Third & $14(28.1)$ & $7(33.3)$ & $7(21.9)$ & \\
\hline Fourth & $37(68.4)$ & $12(57.1)$ & $25(78.1)$ & \\
\hline Internal echogenicity, $n(\%)$ & & & & 0.744 \\
\hline Hyperechoic & $10(18.9)$ & $4(19.0)$ & $6(18.8)$ & \\
\hline Hypoechoic & $31(58.5)$ & $14(66.7)$ & $17(53.1)$ & \\
\hline Anechoic & $1(1.9)$ & $0(0.0)$ & $1(3.1)$ & \\
\hline Mixed & $11(20.7)$ & $3(14.3)$ & $8(25.0)$ & \\
\hline Echotexture, $n(\%)$ & & & & 0.096 \\
\hline Homogeneous & $17(32.1)$ & $10(47.6)$ & $7(21.9)$ & \\
\hline Heterogeneous & $36(67.9)$ & $11(52.4)$ & $25(78.1)$ & \\
\hline
\end{tabular}

${ }^{a)}$ Normally distributed variables are expressed as mean \pm standard deviation (range); ${ }^{\text {b) }}$ Non-normally distributed variables are expressed as median (25th-75th percentiles). 
mean age of patients with malignant/potentially malignant gastric SETs was significantly higher than that of patients with benign SETs ( $52.7 \pm 14.1$ years vs. $65.4 \pm 13.3$ years, $p=0.002$ ). On the basis of histopathology results, the median size of the SETs in all patients was $3.0 \mathrm{~cm}$ (range, 1st quartile, $2.5 \mathrm{~cm}$; 3 rd quartile, $4.5 \mathrm{~cm}$ ), with no difference in the median size between the malignant/potentially malignant and benign gastric SETs $(3.0 \mathrm{~cm})$. The body $(33 / 53,62.3 \%)$ was the most common location of gastric SETs based on EGD, followed by the antrum $(8 / 53,15.1 \%)$, cardia $(7 / 53,13.2 \%)$, and fundus $(5 / 53,9.4 \%)$. For benign gastric SETs, the most common location was the body $(8 / 21,38.1 \%)$, followed by the antrum $(7 / 21,33.3 \%)$ and cardia $(6 / 21,28.6 \%)$. Most leiomyomas were found in the cardia $(6 / 8,75.0 \%)$ (Fig. 1A), whereas ectopic pancreas was commonly detected in the antrum $(4 / 7,57.1 \%)$ and body $(3 / 7$, $42.9 \%$ ) (Fig. 1C). The most common location of malignant/ potentially malignant SETs was the body $(25 / 32,78.1 \%)$, followed by the fundus $(5 / 32,15.6 \%)$. In particular, GISTs were frequently found in the body $(25 / 31,80.6 \%)$ and fundus $(4 / 31$, $12.9 \%$ ) (Fig. 1B). In terms of the layer of origin as identified using EUS, benign gastric SETs originated in the second (2/21, $9.5 \%)$, third (7/21, 33.3\%), and fourth (12/21, 57.1\%) echolayer, whereas malignant/potentially malignant gastric SETs were mostly found in the fourth echolayer $(25 / 32,78.1 \%)$. With respect to echotexture on EUS, benign gastric SETs showed relatively even findings between homogeneous (10/21, 47.6\%)
(A)

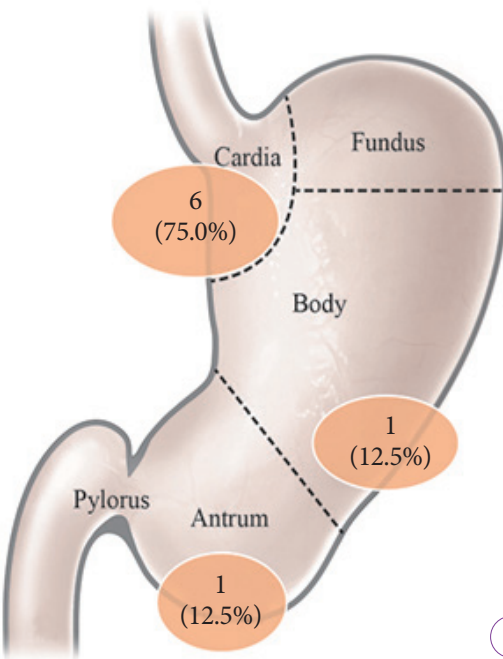

B

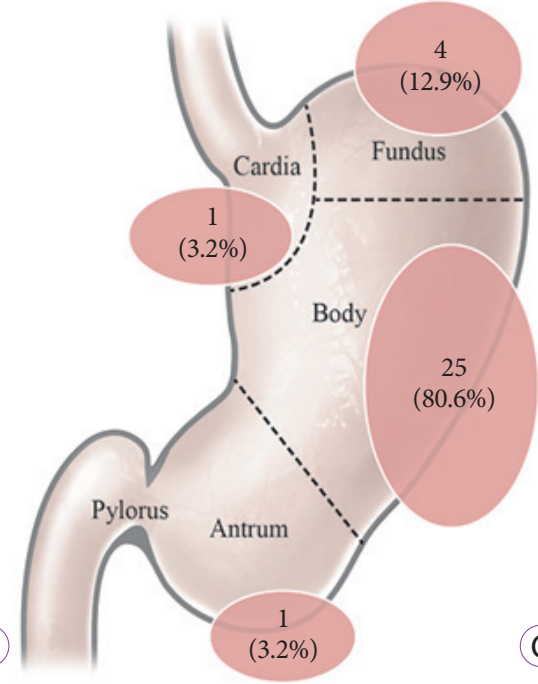

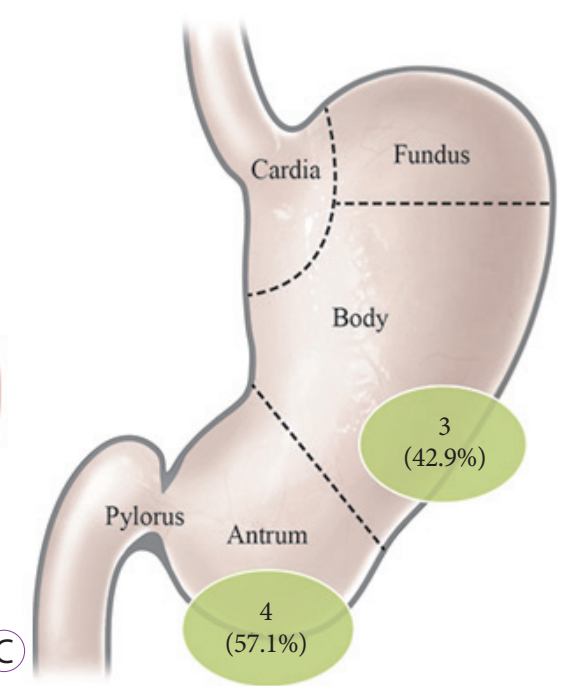

Fig. 1. Distribution of gastric subepithelial tumors: (A) leiomyoma, (B) gastrointestinal stromal tumor, and (C) ectopic pancreas.

Table 3. Accuracy of Endoscopic Ultrasonography and Abdominopelvic Computed Tomography in the Diagnosis of Gastric Subepithelial Tumors

\begin{tabular}{|c|c|c|c|}
\hline Histopathology results & Accuracy of EUS, $n /$ total (\%) & Accuracy of APCT, $n /$ total (\%) & $p$-value \\
\hline Overall gastric SETs & $34 / 53(64.2)$ & $27 / 53(50.9)$ & 0.238 \\
\hline Malignant/Potentially malignant & & & 0.762 \\
\hline GIST & $26 / 31(83.9)$ & $23 / 31(74.2)$ & \\
\hline Lymphoma & $0 / 1(0.0)$ & $1 / 1(100.0)$ & \\
\hline Benign & & & 0.160 \\
\hline Leiomyoma & $3 / 8(37.5)$ & $0 / 8(0.0)$ & \\
\hline Ectopic pancreas & $4 / 7(57.1)$ & $1 / 7(14.3)$ & \\
\hline Schwannoma & $0 / 3(0.0)$ & $0 / 3(0.0)$ & \\
\hline Lipoma & $0 / 1(0.0)$ & $1 / 1(100.0)$ & \\
\hline Gastric duplication cyst & $1 / 1(100.0)$ & $1 / 1(100.0)$ & \\
\hline Adenomyoma & $0 / 1(0.0)$ & $0 / 1(0.0)$ & \\
\hline
\end{tabular}

APCT, abdominopelvic computed tomography; EUS, endoscopic ultrasonography; GIST, gastrointestinal stromal tumor; SET, subepithelial tumor. 
and heterogeneous (11/21, 52.4\%) echotexture; however, a heterogeneous echotexture $(25 / 32,78.1 \%)$ was predominantly observed in malignant/potentially malignant gastric SETs. Overall, significant differences were found in patient age $(p=0.002)$ and tumor location $(p<0.001)$ between benign and malignant/ potentially malignant gastric SETs.

\section{Ability of endoscopic ultrasonography and abdom- inopelvic computed tomography in the diagnosis of gastric subepithelial tumors}

Compared with the surgical histopathology results, the overall accuracy of EUS was $64.2 \%$ (34/53). In detail, the diagnostic accuracy of EUS for GISTs, leiomyomas, ectopic pancreas, and schwannomas was $83.9 \%(26 / 31), 37.5 \%(3 / 8)$, $57.1 \%(4 / 7)$, and $0.0 \%(0 / 3)$, respectively. In contrast, the overall accuracy of APCT was 50.9\% (27/53), with an accuracy for GISTs, leiomyomas, ectopic pancreas, and schwannomas of $74.2 \%(23 / 31), 0.0 \%(0 / 8), 14.3 \%(1 / 7)$, and $0.0 \%(0 / 3)$, respectively (Table 3 ).

For both imaging modalities, the sensitivity for malignant/ potentially malignant gastric SETs was relatively high; however, the specificity was low. In detail, for the diagnosis of malignant/ potentially malignant gastric SETs, EUS had a sensitivity, specificity, PPV, and NPV of $81.3 \%, 38.1 \%, 66.7 \%$, and $57.1 \%$, respec-

Table 4. Diagnostic Yields of Endoscopic Ultrasonography and Abdominopelvic Computed Tomography in the Diagnosis of Malignant/Potentially Malignant Gastric Subepithelial Tumors

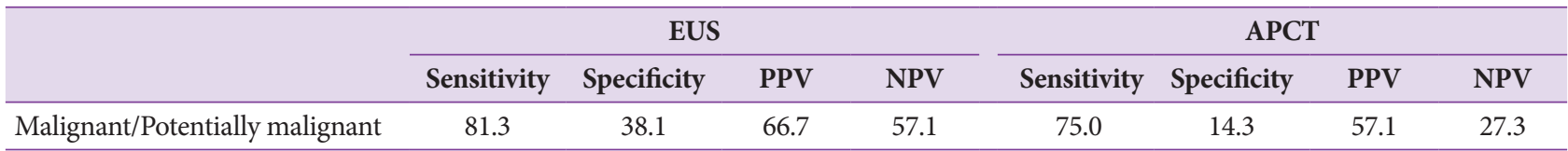

APCT, abdominopelvic computed tomography; EUS, endoscopic ultrasonography; NPV, negative predictive value; PPV, positive predictive value.

Table 5. Characteristics of Misdiagnosed Cases by Endoscopic Ultrasonography Findings Compared to Histopathology Results

\begin{tabular}{|c|c|c|c|c|c|c|c|c|c|c|}
\hline No. & Age & Sex & $\begin{array}{c}\text { EUS } \\
\text { findings }\end{array}$ & $\begin{array}{l}\text { APCT } \\
\text { findings }\end{array}$ & $\begin{array}{c}\text { Histopathology } \\
\text { results }\end{array}$ & $\begin{array}{c}\text { Long } \\
\text { diameter }(\mathrm{cm})\end{array}$ & Location & $\begin{array}{c}\text { Layer of } \\
\text { origin }\end{array}$ & $\begin{array}{c}\text { Internal } \\
\text { echogenity }\end{array}$ & Echotexture \\
\hline 1 & 59 & $\mathrm{~W}$ & GIST & GIST & Leiomyoma & 4.0 & Body & Fourth & Hypoechoic & Homogenous \\
\hline 2 & 75 & W & GIST & Undetected & Leiomyoma & 3.0 & Antrum & Second & Hypoechoic & Heterogenous \\
\hline 3 & 61 & $\mathrm{~W}$ & GIST & Possible $^{\text {a) }}$ & Leiomyoma & 6.0 & Cardia & Fourth & Hypoechoic & Homogenous \\
\hline 4 & 37 & M & GIST & GIST & Leiomyoma & 5.2 & Cardia & Fourth & Hypoechoic & Heterogenous \\
\hline 5 & 52 & $\mathrm{~W}$ & GIST & GIST & Leiomyoma & 5.0 & Cardia & Fourth & Hypoechoic & Heterogenous \\
\hline 6 & 60 & W & GIST & GIST & Ectopic pancreas & 0.8 & Body & Fourth & Hypoechoic & Homogenous \\
\hline 7 & 36 & $\mathrm{~W}$ & GIST & Undetected & Ectopic pancreas & 1.3 & Antrum & Third & Hypoechoic & Homogenous \\
\hline 8 & 70 & $\mathrm{~W}$ & GIST & Possible & Schwannoma & 3.5 & Body & Fourth & Hypoechoic & Homogenous \\
\hline 9 & 65 & $\mathrm{~W}$ & GIST & GIST & Schwannoma & 2.8 & Body & & Hypoechoic & Homogenous \\
\hline 10 & 54 & W & GIST & Lipoma & Lipoma & 4.5 & Antrum & Third & Hyperechoic & Heterogenous \\
\hline 11 & 77 & $\mathrm{~W}$ & Leiomyoma & GIST & GIST & 2.6 & Body & Fourth & Hypoechoic & Homogenous \\
\hline 12 & 53 & W & Leiomyoma & GIST & GIST & 1.4 & Body & Fourth & Hypoechoic & Homogenous \\
\hline 13 & 48 & $\mathrm{~W}$ & Leiomyoma & GIST & GIST & 2.5 & Body & Fourth & Hypoechoic & Homogenous \\
\hline 14 & 58 & $\mathrm{~W}$ & Leiomyoma & GIST & GIST & 3.0 & Fundus & & Hypoechoic & Homogenous \\
\hline 15 & 60 & M & Leiomyoma & GIST & GIST & 1.7 & Body & Fourth & Hypoechoic & Heterogenous \\
\hline 16 & 68 & W & Leiomyoma & Possible $^{\text {a) }}$ & Schwannoma & 3.5 & Body & Fourth & Hypoechoic & Homogenous \\
\hline 17 & 72 & M & GCP & Lymphoma & Lymphoma & 3.0 & Body & Third & Hypoechoic & Heterogenous \\
\hline 18 & 53 & M & $\begin{array}{l}\text { Ectopic } \\
\text { pancreas }\end{array}$ & Undetected & Adenomyoma & 1.8 & Antrum & Third & Hyperechoic & Homogenous \\
\hline 19 & 48 & $\mathrm{~W}$ & lipoma & GIST & Ectopic pancreas & 5.2 & Body & Third & Hyperechoic & Heterogenous \\
\hline
\end{tabular}

APCT, abdominopelvic computed tomography; EUS, endoscopic ultrasonography; GCP, gastritis cystica profunda; GIST, gastrointestinal stromal tumor.

a) Type of gastric subepithelial tumors was not clearly specified due to limitation of resolution capability on APCT images. 
tively, whereas APCT had a sensitivity, specificity, PPV, and NPV of $75.0 \%, 14.3 \%, 57.1 \%$, and $27.3 \%$, respectively (Table 4 ).

\section{Cases of misdiagnosed gastric subepithelial tumors with endoscopic ultrasonography}

Table 5 presents representative cases of misdiagnosed gastric SETs. We compared the presumptive diagnosis using EUS with the definitive diagnosis using histopathology after laparoscopic wedge resection. The investigators who analyzed the EUS findings identified 34 of the 53 cases as malignant/ potentially malignant gastric SETs. Of these, 10 cases, which were mostly misdiagnosed as GISTs, were proven to be benign gastric SETs, including leiomyoma $(n=5)$, ectopic pancreas $(n=2)$, schwannoma $(n=2)$, and lipoma $(n=1)$. In particular, if hypoechoic lesions originating in the fourth echolayer were observed on the cardia, the EUS investigators commonly misdiagnosed leiomyoma as GIST (Fig. 2). Conversely, all 5 GISTs were misdiagnosed as leiomyomas with EUS.

\section{DISCUSSION}

Because EUS can provide precise internal images of the layer of origin, it has been recognized as the most efficient diagnostic modality for gastric SETs. ${ }^{1,6}$ However, APCT has other advantages in evaluating the characteristics, local extension, invasion to adjacent organs, or possible metastasis of SETs. ${ }^{9}$ Because of the different benefits of the 2 modalities, we evaluated the diagnostic ability of EUS and APCT for gastric SETs based on surgical histopathology results.

Consistent with previously reported results, we found that the overall accuracy of EUS in diagnosing gastric SETs was $64.2 \% .{ }^{15}$ In comparison, APCT had an overall accuracy of $50.9 \%$, which is somewhat lower than that of EUS. Both EUS and APCT demonstrated good results for the detection of GISTs in this study. Further, although both modalities exhibited low diagnostic accuracy for most benign gastric SETs, EUS was more reliable for leiomyomas and ectopic pancreas,
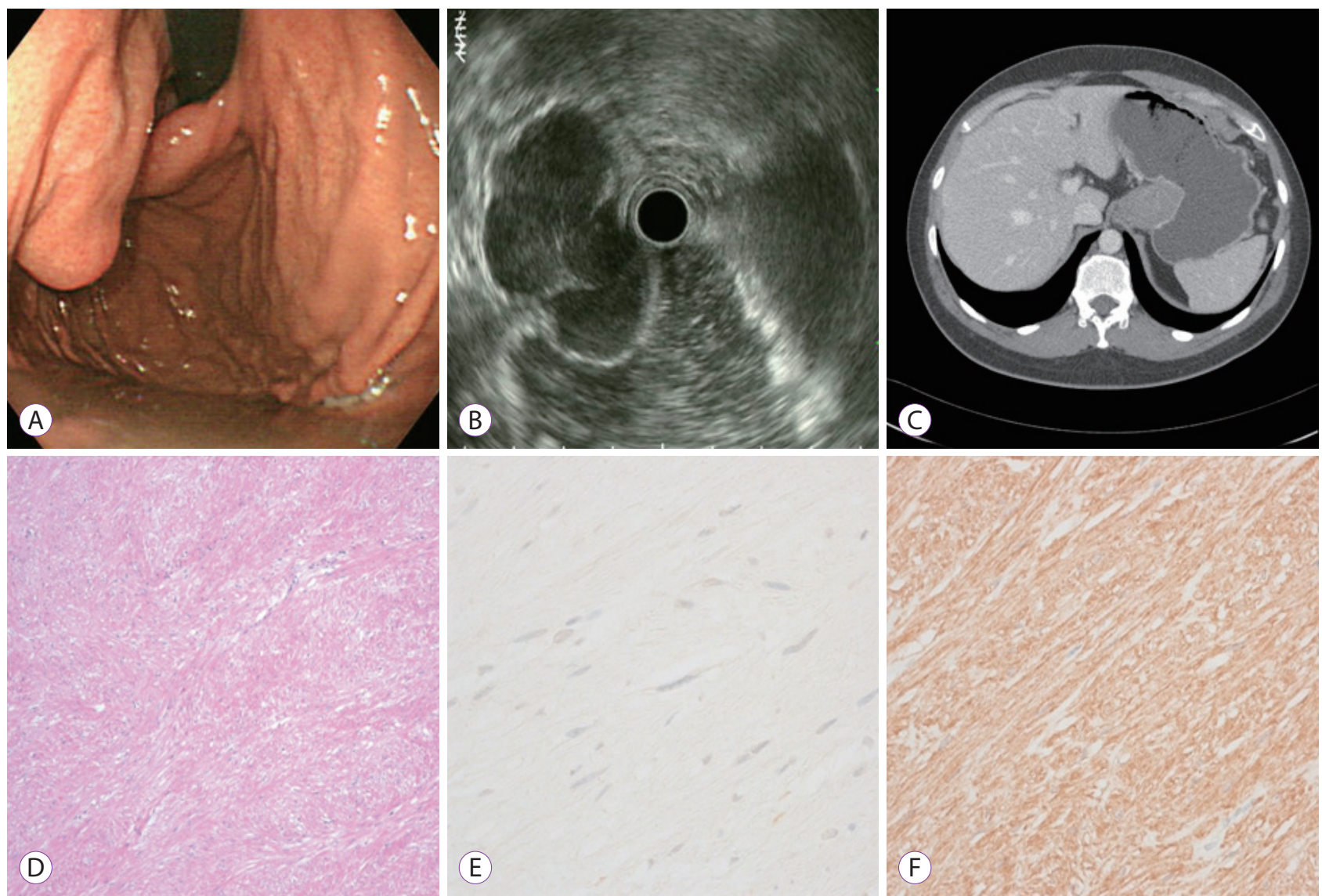

Fig. 2. Leiomyoma was misdiagnosed as a gastrointestinal stromal tumor (GIST) based on endoscopic ultrasonography (EUS) findings in a 37-year-old man (Table 4, case 4). (A) Upper endoscopy showed a gastric subepithelial tumor with a size of about $5.0 \mathrm{~cm}$ on the cardia. (B) EUS showed a $5.2-\mathrm{cm}$, well-defined, hypoechoic with a heterogeneous echotexture, and septated lesion mainly originating in the fourth layer. The lesion was presumed to be a GIST. (C) Contrast-enhanced computed tomography axial image showed a $5.1-\mathrm{cm}$, well-demarcated, and outward-protruding mass with homogeneous enhancement on the cardia. The lesion was presumed to be a GIST. (D) The histopathological result of surgical resection was leiomyoma with a size of $5.2 \mathrm{~cm}$, which was composed of spindle cells as observed on hematoxylin and eosin staining $(\times 10)$. (E) Immunohistochemical staining was negative for $c-k i t(\times 40)$. (F) Immunohistochemical staining was positive for actin $(\times 40)$. 
whereas APCT was more acceptable for lipomas and GDCs.

On EUS, GISTs are generally characterized as hypoechoic or having mixed echogenicity, having a homogeneous echotexture, and mainly originating in the fourth echolayer (corresponding to the muscularis propria). ${ }^{6,16}$ In addition, GISTs with high-risk features might present various EUS findings such as irregular shape, heterogeneous echotexture, echogenic foci, central necrosis, or calcification. ${ }^{17}$ In this study, 7 GISTs exhibited a homogeneous echotexture and 24 had a heterogeneous echotexture and various echogenicities including hyperechoic, hypoechoic, or mixed with echogenic foci or central necrosis. Most of these EUS findings closely corresponded with those of previous reports. ${ }^{6,16}$ On APCT, GISTs usually present with a well-defined margin and homogeneous contrast enhancement. ${ }^{14}$ Previous studies have reported that attenuation values of 30-35 HU in pre-contrast imaging and 50-60 HU in post-contrast imaging are helpful in the differential diagnosis of GISTs using APCT. ${ }^{10,18}$ APCT may also identify differences in appearance and enhancement based on the size of the GIST; in particular, for GISTs $>5 \mathrm{~cm}$, an ill-defined margin and heterogeneous enhancement with central necrosis or calcification were identifiable. In our study, 14 GISTs had a smooth margin and homogeneous contrast enhancement and 9 had an ill-defined margin and heterogeneous enhancement. It was also found that 8 GISTs had low or indefinite enhancement.

Hypoechoic echogenicity and diffuse thickening with fusion of the echolayers are usually reported as the key EUS features for diagnosing lymphoma. ${ }^{19,20}$ However, as these are not always apparent in EUS, an accurate diagnosis is difficult with EUS alone. In the present study, EUS revealed a thickening of the third layer with multiple anechoic areas. Consequently, we identified lymphoma as gastritis cystica profunda. On APCT, lymphoma exhibits various imaging features, including single or multiple masses with or without ulcers, thickened folds, and mucosal nodularity of varying sizes, ${ }^{21}$ which are characteristics similar to those exhibited by gastric carcinoma. A previous study demonstrated that the following features can strongly suggest a lymphoma: a bulky mass or diffuse infiltration with preservation of the fat planes, involvement of multiple sites, and lymphadenopathy. ${ }^{22}$ In our study, gastric wall thickening with preservation of the overlying mucosa and an accompanying lymphadenopathy were identified using APCT, which we considered possible indications of a lymphoma.

For benign gastric SETs, EUS is a more accurate and reliable diagnostic modality than APCT. Particularly for leiomyoma and ectopic pancreas, EUS may reveal subtle findings not seen with APCT. With EUS, leiomyomas can be characterized as well circumscribed, with a homogeneous echotexture and hypoechoic echogenicity and mainly originating in either the fourth or the second echolayer (corresponding to the muscularis mucosa). ${ }^{6}$ In this study, the presumptive EUS diagnosis was correct for only 3 of the 8 leiomyoma cases because many of the EUS features associated with leiomyomas overlap with those of GISTs. Ectopic pancreas usually exhibits a heterogeneous echotexture and an anechoic ductal structure, while mostly developing in either the third (corresponding to the submucosa) or fourth echolayer. ${ }^{23}$ In our study, 3 cases of ectopic pancreas originated in the fourth echolayer, 3 cases in the third echolayer, and 1 case in the second echolayer. Moreover, 3 cases of ectopic pancreas were characterized by a mixed echogenicity and an anechoic ductal structure, whereas 4 exhibited a hypoechoic echogenicity. However, the accuracy of APCT was not satisfactory for either leiomyoma or ectopic pancreas. In the present study, APCT did not detect leiomyoma in $12.5 \%(1 / 8)$ and ectopic pancreas in $28.6 \%(2 / 7)$ of the patients because small lesions were obscured. Owing to the limited resolution of CT images, the 2 radiologists did not identify leiomyoma in $12.5 \%(1 / 8)$ and ectopic pancreas in $14.3 \%(1 / 7)$ of the patients. Although APCT features for benign gastric SETs are generally nonspecific, several studies have reported that leiomyoma and ectopic pancreas exhibit some characteristic CT features. ${ }^{11,12,24,25}$ In a study comparing CT findings with pathologically proven gastric true leiomyoma, leiomyoma is homogeneous, has poor-to-moderate enhancement, and has low attenuation or iso-attenuation compared with muscle attenuation. ${ }^{25}$ However, we found that APCT did not provide valuable features for identifying leiomyomas to the same extent as it did for GISTs. In the diagnosis of ectopic pancreas, a previous study demonstrated that an antrum location, ill-defined margin, prominent enhancement of the overlying mucosa, and long diameter/short diameter ratio of $>1.4$ are specific APCT criteria. ${ }^{11}$ However, in the present study, 3 of the 7 ectopic pancreas cases were located in the body and 6 cases did not show a specific enhancement pattern.

On the other hand, the accuracy of APCT was acceptable for lipoma and GDC in this study. Lipoma typically presents with a well-circumscribed submucosal mass with uniform fat attenuation (70-120 HU), allowing a conclusive diagnosis to be made using APCT. ${ }^{23,26}$ In addition, GDC can be diagnosed using APCT based on a well-defined, homogeneous, thickwalled cystic lesion with an enhanced inner lining, occasionally accompanied by calcification. These features have also been shown to be of diagnostic value for GDC in another study. ${ }^{27}$ Although APCT did not demonstrate satisfactory accuracy for benign gastric SETs, it can play a complementary role in providing additional characteristics for lipomas and GDCs.

We classified cases in which there was a discrepancy between the histopathology results and preoperative EUS diag- 
noses based on size, location, layer of origin, internal echogenicity, and echotexture. Most of these discrepancies involved hypoechoic lesions originating in the fourth echolayer in the body or cardia. These characteristics are commonly detected in mesenchymal tumors, including GISTs, leiomyomas, and schwannomas; thus, accurate diagnosis may be not easy in these cases. When gastric SETs are located in the cardia, access of the EUS probe is difficult. Consequently, the origin layer and internal echogenicity may not be clear and distinct in the cardia. In the present study, GISTs were commonly misdiagnosed as leiomyomas, and leiomyomas were commonly misdiagnosed as GISTs with EUS. Even when the size was $>5$ $\mathrm{cm}$, a leiomyoma located in the cardia was often mistakenly assumed to be a GIST. Predicting the possibility of malignancy and making a clinical decision of whether to observe the progress of the lesion, to perform EUS-guided biopsy, or to choose resection are important. ${ }^{16,28}$ In particular, laparoscopic wedge resection cannot be easily applied to gastric SETs located in the cardia because of the technical complexities of the procedure. ${ }^{29}$ These cases require invasive surgery and have a high risk of gastro-esophageal reflux as a complication. To avoid unnecessary surgery, EUS-guided biopsy should be considered when gastric SETs in the cardia exhibit malignant EUS features.

Previous studies have reported that the location, layer of origin, and shape of SETs can be used to predict their type. ${ }^{30}$ Our data revealed specific patterns for the location of gastric SETs that were similar to those reported in previous studies. ${ }^{30-32}$ If a lesion is located in the cardia, the chance that it is a leiomyoma is high. GISTs are commonly located in the body and fundus, whereas ectopic pancreas is mainly located in the antrum. Although it cannot be applied to all types of gastric SETs, tumor location could be used to narrow down the differential diagnosis and could even be diagnostic in some cases.

Our study has some limitations. First, the potential for selection bias was present, as we performed a retrospective analysis and included only surgically resected cases. Second, the study was conducted at a single center; hence, interobserver variation could not be assessed. Third, the number of cases was relatively small. Therefore, a prospective study that includes a larger sample from multiple institutions is required to confirm our results. Finally, the 2 radiologists were blinded to the patients' clinical information such as EGD findings. If the patients' clinical background had been provided to the radiologists, the diagnostic accuracy of APCT could have been improved.

In conclusion, we investigated the diagnostic ability of EUS and APCT for gastric SETs relative to the conclusive diagnosis based on the surgical histopathology results. APCT exhibited lower overall accuracy than EUS. Nevertheless, APCT is a meritorious modality for malignant/potentially malignant gastric SETs including GISTs and lymphomas. APCT could also have a complementary role in providing valuable information on some benign gastric SETs such as lipomas and GDCs. For hypoechoic lesions originating in the fourth echolayer, EUS imaging alone is insufficient to accurately diagnose gastric SETs, although the location of gastric SETs could help in the differential diagnosis.

\section{Conflicts of Interest}

The authors have no financial conflicts of interest.

\section{Author Contributions}

Conceptualization: Sang Yoon Kim, Ki-Nam Shim, Joo-Ho Lee, HyeKyung Jung, Chang Mo Moon, Seong-Eun Kim, Sung-Ae Jung

Data curation: SYK, KNS, JHL, Ji Young Lim, Tae Oh Kim, A. Reum Choe, Chung Hyun Tae

Formal analysis: SYK, KNS

Investigation: SYK, KNS

Methodology: SYK, KNS

Writing-original draft: SYK

Writing-review\&editing: KNS

\section{REFERENCES}

1. Hwang JH, Rulyak SD, Kimmey MB. American Gastroenterological Association institute technical review on the management of gastric subepithelial masses. Gastroenterology 2006;130:2217-2228.

2. Humphris JL, Jones DB. Subepithelial mass lesions in the upper gastrointestinal tract. J Gastroenterol Hepatol 2008;23:556-566.

3. Hedenbro JL, Ekelund M, Wetterberg P. Endoscopic diagnosis of submucosal gastric lesions. The results after routine endoscopy. Surg Endosc 1991;5:20-23.

4. Lee DH, Ko YT. Gastric lesions: evaluation with three-dimensional images using helical CT. AJR Am J Roentgenol 1997;169:787-789.

5. Kim SY, Kim KO. Management of gastric subepithelial tumors: the role of endoscopy. World J Gastrointest Endosc 2016;8:418-424.

6. Sakamoto H, Kitano M, Kudo M. Diagnosis of subepithelial tumors in the upper gastrointestinal tract by endoscopic ultrasonography. World J Radiol 2010;2:289-297.

7. Chak A. EUS in submucosal tumors. Gastrointest Endosc 2002;56(4 Suppl):S43-S48.

8. Kim JH, Eun HW, Goo DE, Shim CS, Auh YH. Imaging of various gastric lesions with 2D MPR and CT gastrography performed with multidetector CT. Radiographics 2006;26:1101-1116; discussion 1117-1118.

9. Ra JC, Lee ES, Lee JB, et al. Diagnostic performance of stomach CT compared with endoscopic ultrasonography in diagnosing gastric subepithelial tumors. Abdom Radiol (NY) 2017;42:442-450.

10. Lee CM, Chen HC, Leung TK, Chen YY. Gastrointestinal stromal tumor: computed tomographic features. World J Gastroenterol 2004;10:2417-2418.

11. Kim JY, Lee JM, Kim KW, et al. Ectopic pancreas: CT findings with emphasis on differentiation from small gastrointestinal stromal tumor and leiomyoma. Radiology 2009;252:92-100.

12. Megibow AJ, Balthazar EJ, Hulnick DH, Naidich DP, Bosniak MA. CT evaluation of gastrointestinal leiomyomas and leiomyosarcomas. AJR Am J Roentgenol 1985;144:727-731.

13. Goto O, Kambe H, Niimi K, et al. Discrepancy in diagnosis of gastric 
submucosal tumor among esophagogastroduodenoscopy, CT, and endoscopic ultrasonography: a retrospective analysis of 93 consecutive cases. Abdom Imaging 2012;37:1074-1078.

14. Okten RS, Kacar S, Kucukay F, Sasmaz N, Cumhur T. Gastric subepithelial masses: evaluation of multidetector CT (multiplanar reconstruction and virtual gastroscopy) versus endoscopic ultrasonography. Abdom Imaging 2012;37:519-530.

15. Lim TW, Choi CW, Kang DH, Kim HW, Park SB, Kim SJ. Endoscopic ultrasound without tissue acquisition has poor accuracy for diagnosing gastric subepithelial tumors. Medicine (Baltimore) 2016;95:e5246.

16. Moon JS. Role of endoscopic ultrasonography in guidingtreatment plans for upper gastrointestinal subepithelial tumors. Clin Endosc 2016;49:220-225.

17. Kang JH, Lim JS, Kim JH, et al. Role of EUS and MDCT in the diagnosis of gastric submucosal tumors according to the revised pathologic concept of gastrointestinal stromal tumors. Eur Radiol 2009;19:924-934.

18. Ludwig DJ, Traverso LW. Gut stromal tumors and their clinical behavior. Am J Surg 1997;173:390-394

19. Caletti G, Fusaroli P, Togliani T, Bocus P, Roda E. Endosonography in gastric lymphoma and large gastric folds. Eur J Ultrasound 2000;11:3140.

20. Schizas D, Ntanasis-Stathopoulos I, Tsilimigras DI, et al. The role of endoscopic ultrasound in the diagnosis and management of primary gastric lymphoma. Gastroenterol Res Pract 2017;2017:2397430.

21. Gossios K, Katsimbri P, Tsianos E. CT features of gastric lymphoma. Eur Radiol 2000;10:425-430.

22. Ghai S, Pattison J, Ghai S, O’Malley ME, Khalili K, Stephens M. Primary gastrointestinal lymphoma: spectrum of imaging findings with patho- logic correlation. Radiographics 2007;27:1371-1388.

23. Lin YM, Chiu NC, Li AF, Liu CA, Chou YH, Chiou YY. Unusual gastric tumors and tumor-like lesions: radiological with pathological correlation and literature review. World J Gastroenterol 2017;23:2493-2504.

24. Cho JS, Shin KS, Kwon ST, et al. Heterotopic pancreas in the stomach: CT findings. Radiology 2000;217:139-144.

25. Lee MJ, Lim JS, Kwon JE, et al. Gastric true leiomyoma: computed tomographic findings and pathological correlation. J Comput Assist Tomogr 2007;31:204-208.

26. Taylor AJ, Stewart ET, Dodds WJ. Gastrointestinal lipomas: a radiologic and pathologic review. AJR Am J Roentgenol 1990;155:1205-1210.

27. Maeda H, Okabayashi T, Nishimori I, et al. Diagnostic challenge to distinguish gastric duplication cyst from pancreatic cystic lesions in adult. Intern Med 2007;46:1101-1104.

28. Shin SY, Lee SJ, Jun JH, et al. Mucosal incision and forceps biopsy for reliable tissue sampling of gastric subepithelial tumors. Clin Endosc 2017;50:64-68.

29. Lee JS, Kim JJ, Park SM. Laparoscopic gastric wedge resection and prophylactic antireflux surgery for a submucosal tumor of gastroesophageal junction. J Gastric Cancer 2011;11:131-134.

30. Seo SW, Hong SJ, Han JP, et al. Accuracy of a scoring system for the differential diagnosis of common gastric subepithelial tumors based on endoscopic ultrasonography. J Dig Dis 2013;14:647-653.

31. Kim DJ, Lee JH, Kim W. Laparoscopic resection for 125 gastroduodenal submucosal tumors. Ann Surg Treat Res 2014;86:199-205.

32. Lee HH, Hur H, Jung H, Jeon HM, Park CH, Song KY. Analysis of 151 consecutive gastric submucosal tumors according to tumor location. J Surg Oncol 2011;104:72-75. 